\title{
Minority Carrier Lifetime Measurements on 4H-SiC Epiwafers by Time- Resolved Photoluminescence and Microwave Detected Photoconductivity
}

\author{
Jan Beyer ${ }^{1, a^{*}}$, Nadine Schüler ${ }^{2, b}$, Jürgen Erlekampf ${ }^{3}$, Birgit Kallinger ${ }^{3}$, \\ Patrick Berwian ${ }^{3}$, Kay Dornich ${ }^{2}$, Johannes Heitmann ${ }^{1}$
}

${ }^{1}$ TU Bergakademie Freiberg, Institute of Applied Physics, Leipziger Str. 23, 09599 Freiberg, Germany

${ }^{2}$ Freiberg Instruments GmbH, Delfter Straße 6, 09599 Freiberg, Germany

${ }^{3}$ Fraunhofer IISB, Schottkystraße 10, 91058 Erlangen, Germany

ajan.beyer@physik.tu-freiberg.de, bschueler@freiberginstruments.com,

Keywords: carrier lifetime, photoluminescence, microwave detected photoconductivity, temperature dependence

\begin{abstract}
Temperature dependent microwave detected photoconductivity MDP and time-resolved photoluminescence TRPL were employed to investigate the carrier lifetime in CVD grown $4 \mathrm{H}-\mathrm{SiC}$ epilayers of different thickness. The minority carrier lifetime may be found from both the MDP and defect PL decay at room temperature for all epilayers, whereas the near bandedge emission (NBE) decay is much faster for thin epilayers $(<17 \mu \mathrm{m})$ due to the substrate proximity and only follows the minority carrier lifetime for thicker samples at lower excess carrier concentrations.
\end{abstract}

\section{Introduction}

The minority carrier lifetime is one of the fundamental parameters with regard to the performance of semiconductor devices, especially for the application of $\mathrm{SiC}$ in high voltage devices. Hence, carrier lifetime engineering is an appropriate way to gain best performance of devices for different applications. Different temperature treatments increase or decrease the concentration of carbon vacancies forming the $\mathrm{Z}_{1 / 2}$ defect [1], which is the main "lifetime killer" in $4 \mathrm{H}-\mathrm{SiC}$. For epitaxial layers, however, it is complex to determine the main influence on the lifetime, as the epilayer surface, the epilayer-substrate interface, the epilayer and the substrate contribute to carrier recombination [2]. Furthermore, measurement conditions, such as e.g. the excitation wavelength and intensity, play an important role for the observed effective carrier lifetime. This contribution aims to contribute to a better understanding of this problem by comparing time constants of photoluminescence and photoconductivity decay measurements obtained under the same excitation conditions for $4 \mathrm{H}-\mathrm{SiC}$ epitaxial layers of different thicknesses and at different sample temperatures.

\section{Samples and Methods}

$4 \mathrm{H}-\mathrm{SiC}$ epilayers with thicknesses ranging from 12 to $62 \mu \mathrm{m}$ (determined by FTIR), grown on $350 \mu \mathrm{m}$ thick $4 \mathrm{H}-\mathrm{SiC} \mathrm{n}^{+}$substrates (resistivity about $0.02 \Omega \cdot \mathrm{cm}$ ) by chemical vapor deposition [3] were investigated. All epilayers were n-type doped at $10^{14}$ to low $10^{15} \mathrm{~cm}^{-3}$.

Microwave detected photoconductivity measurements (MDP, carried out with a Freiberg Instruments MDPmap) as well as time-resolved PL measurements (TRPL) were performed under high injection conditions, excited by $3 \mathrm{~ns}$ pulses from the same nitrogen laser $(337 \mathrm{~nm}, 10-50 \mu \mathrm{J}$ per pulse corresponding to injection levels of about 1 to $5 \cdot 10^{17} \mathrm{~cm}^{-3}$ ) with a penetration depth of $14 \mu \mathrm{m}$. For thicker epilayers, most of the incident light is absorbed in the epilayer and only few percent reaches the substrate, e.g. $2 \%$ for a $55 \mu \mathrm{m}$ thick epilayer. For thinnest layers $(12 \mu \mathrm{m})$, only $58 \%$ of the incident light is absorbed in the epilayer. Separate PL experiments on the substrate showed that 
it is of sufficient quality to emit a PL spectrum roughly similar to the epilayer spectrum, but with decay time constants consistently at or below the time resolution of our measurement setup ( $\approx 7 \mathrm{~ns})$.

The MDP microwave (with a frequency of $9.4 \mathrm{GHz}$ ), penetrates the epilayer very well (even for $10^{17} \mathrm{~cm}^{-3}$ excited charge carriers, the skin depth is $137 \mu \mathrm{m}$ ), but due to the low resistivity of the substrate, the skin depth there is reduced to around $70 \mu \mathrm{m}$.

Effects of carrier diffusion will be limited to the very initial stages of the observed transients as both the lateral extent of the illuminated area $\left(1.9 \times 3.5 \mathrm{~mm}^{2}\right)$ is much larger than the diffusion length and the depth diffusion of carriers in conjunction with surface and substrate recombination will lead to a roughly time-invariant carrier concentration profile shape within a very short time $[4,5]$.

\section{Results and Discussion}

Typical room temperature decay curves of MDP and PL measurements on a $55 \mu \mathrm{m}$ thick epilayer are shown in Fig. 1. The near-bandedge emission (NBE) decay (detected at $391 \mathrm{~nm}$ ) showed a double exponential decay, where the slower component is typically ascribed to the minority carrier lifetime [6,7]. The defect-related PL band at $510 \mathrm{~nm}$ showed a more complex multi-exponential decay behavior and is typically reported to be related either to residual boron $[8,9]$ or structural defects $[10,11]$. The MDP signal typically showed a single exponential decay, influenced by both electron and hole contributions [7]. The slower rise time is due to a longer response time constant of the current MDP detection electronics ( $\approx 70 \mathrm{~ns})$ compared to the TRPL setup ( $\approx 7 \mathrm{~ns})$.

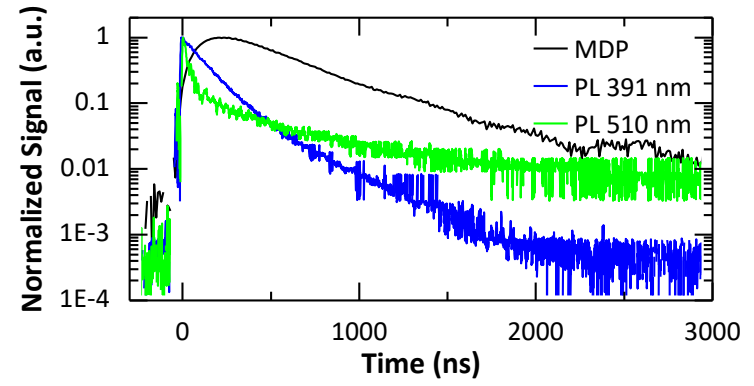

Fig. 1. Normalized signals of MDP (black trace) and PL (blue: NBE at $391 \mathrm{~nm}$; green: defect PL at $510 \mathrm{~nm}$ ) measurements on a $55 \mu \mathrm{m}$ thick $4 \mathrm{H}-\mathrm{SiC}$ epilayer after optical excitation by a 3 ns nitrogen laser pulse.

Room temperature TRPL-MDP correlation. A number of epilayers of varying thickness have been investigated, whereof the layers with a thickness $d>22 \mu \mathrm{m}$ have been found to show a tendency towards direct correlation between the second (longer) component of the NBE PL lifetime and the MDP lifetime, as shown in Fig. 2(a). This hints to the fact that in the investigated samples even the MDP decay is dominated by the minority carrier lifetime. We note that the lifetime values represent effective values, which are in their absolute value still strongly influenced by surface and substrate recombination contributions due to the limited epilayer thickness. As the surface in all cases is in the same as-grown state, the surface recombination velocity will be similar for all the samples. Furthermore, the very short recombination lifetime in the substrate will not contribute to possible lifetime differences between the samples more than as acting as an efficient carrier sink of variable distance (epilayer thickness). Thus, our time constant values are to be taken as a measure proportional to the epilayer carrier lifetime, despite being not exactly the carrier lifetime.

In contrast, "thin" layers with $d<17 \mu \mathrm{m}$ showed a different character of the NBE decay transients. Following an initial sharp drop of varying weight, the main part decayed with a rather short time constant of about $150 \mathrm{~ns}$. The MDP lifetimes were still in the same range as for the thicker epilayers (see Fig. 2(b)). As pointed out above, almost half of the total carriers in these samples are excited in the substrate, which shows fast recombination dynamics. Re-absorption losses of the emitted PL should be small (already the NBE has a penetration depth of about $600 \mu \mathrm{m}$ [12]), so the PL is collected equally well from both the epilayer and the substrate. Consequently, the detected NBE PL signal shows a complex superposition of epilayer and substrate contributions [13]. The MDP signal on the other hand continues to be strongly dominated by the epilayer characteristics 
due to its proportionality to the product of optically excited excess carrier concentration and mobility [7], with the latter one being typically very low in the substrates.
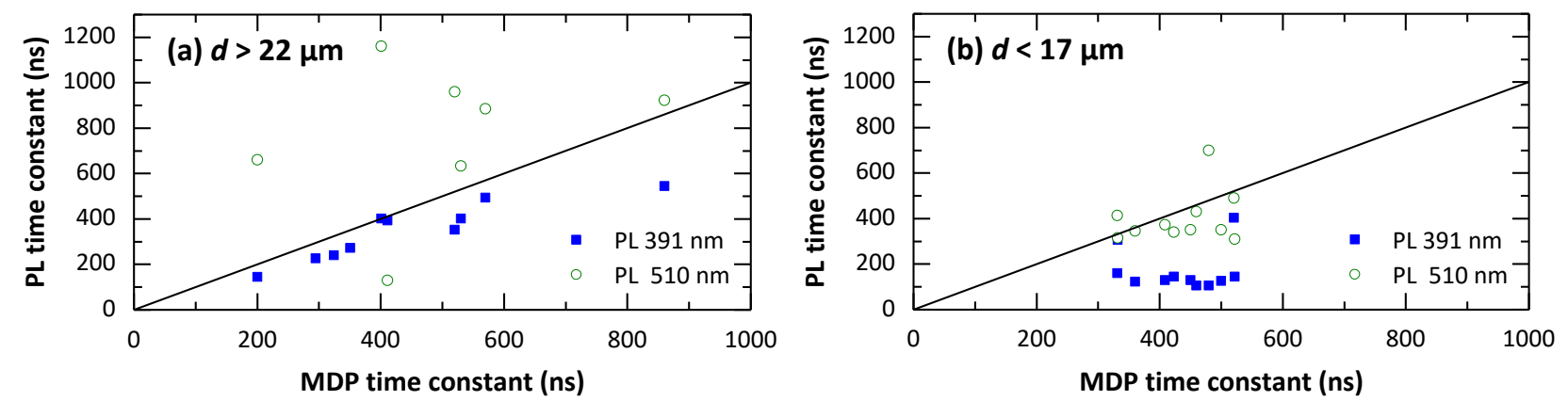

Fig. 2. Correlation of room temperature PL decay time constants (blue squares: $391 \mathrm{~nm}$ PL (NBE) longer decay time; green circles: $510 \mathrm{~nm}$ defect PL longer decay time) to MDP decay time constants for several (a) "thick" (11 samples of mainly $55 \mu \mathrm{m}$ thickness, but also including those of 22.4 to $62.2 \mu \mathrm{m}$ ) and (b) "thin" (11 samples of thicknesses ranging from 11 to $16 \mu \mathrm{m}$, most of them having either 12 or $15 \mu \mathrm{m}) 4 \mathrm{H}-\mathrm{SiC}$ epilayers. The diagonal line is a guide to the eye indicating 1:1 correlation between PL and MDP time constants.

Interestingly, for thin epilayers (the asymptotic part of) the defect PL decay time matched much better to the MDP lifetime, as shown by green open circles in Fig. 2, suggesting that the defect PL decay might be limited by the same recombination mechanism and corresponding lifetime as the MDP signal decay, probably the minority carrier lifetime.

Temperature dependence of carrier lifetime for thick epilayers. To gain deeper insight into the carrier dynamics in these samples, we conducted temperature dependent TRPL and MDP experiments on selected samples - a thick epilayer of $55 \mu \mathrm{m}$ and a thin epilayer of $12 \mu \mathrm{m}$. For the thick epilayer sample, transients and temperature dependent lifetimes are shown in Fig. 3(a).
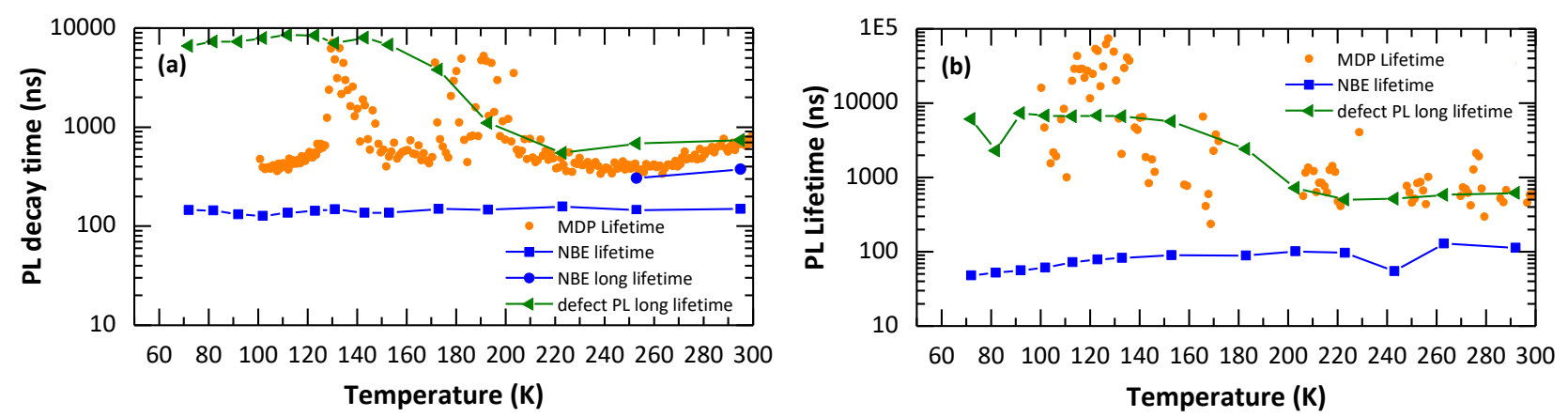

Fig. 3. Temperature dependence of MDP and PL decay time constants for an (a) $55 \mu \mathrm{m}$ and (b) $12 \mu \mathrm{m}$ epilayer.

In the investigated temperature range from $72-293 \mathrm{~K}$, the NBE signal decays predominantly with a temperature-independent single exponential decay time of about $150 \mathrm{~ns}$. Only for the highest temperatures $T>250 \mathrm{~K}$, the slower second contribution, following the minority carrier lifetime, starts to gain relative weight. As typical excitonic lifetimes are much shorter [6], our faster main component rather seems to be due to carrier recombination at the surface or in the substrate [13].

The longest time constant of the defect PL decay shows multi- $\mu$ s values at low temperatures and a thermally activated lifetime quenching. For $T>220 \mathrm{~K}$ the time constant apparently stabilizes at 500-700 ns. This is because the transient fitting routine now tracks the next component of the defect PL decay, which shows only a very slight temperature dependence.

Temperature-dependent MDP showed two-component decay behavior at low temperatures - a main component with a time constant in the 500-700 ns range, rising only slowly at elevated temperatures and a longer one, varying strongly with temperature. The shorter time constant may be dominated by the minority carrier lifetime, matching approximately the longer NBE decay 
component and the slowest defect PL decay at higher temperatures. The longer MDP decay component can be attributed to carrier trapping and thermally activated re-emission effects. Note, that the plotted MDP decay time constants represent single exponential fits covering the intermediate part of the transients and thus vary between the short and long component according to their relative weight, although in the raw data, the fast component is visible continuously.

Temperature dependence of carrier lifetime for thin epilayers. For the thin epilayer, the determined decay time constants are shown in Fig. 3(b). The NBE decay shows mostly multiexponential behavior with a dominating time constant around or below $100 \mathrm{~ns}$ at low temperature. With increasing temperature, an initial sharp drop in the NBE transient gains weight (with a time constant at the system resolution limit). Both should be a consequence of thermally activated carrier diffusion leading to a relative increase in the contribution of carrier recombination in the substrate and at the surface.

The MDP decay follows the same temperature dependent characteristic as for the thick layers, although signal-to-noise ratio is reduced due to the smaller contributing sample volume. Also the defect PL for the thin epilayer is similar in shape and time constants to the thick epilayer. Probably, the defect is limited to the epilayer, so its decay follows the epilayer carrier lifetime.

\section{Summary}

We investigated CVD grown n-type $4 \mathrm{H}-\mathrm{SiC}$ epilayers of varying thickness on $\mathrm{n}^{+} 4 \mathrm{H}-\mathrm{SiC}$ substrates using temperature dependent microwave detected photoconductivity MDP and timeresolved photoluminescence TRPL. MDP signal decay is generally composed of two contributions a fast decay that in our case seems to follow predominantly the minority carrier lifetime and a slow component resulting from carrier trapping and re-emission, the latter being strongly temperature dependent. Surface and substrate influences on the observed carrier concentration decays are strong, due to generally low epilayer thickness. Yet, variations between the samples follow variations in the epilayer's minority carrier lifetime and may be found for the thicker epilayers ( $>22$ to $62 \mu \mathrm{m}$ ) from the near bandedge PL (NBE) decay curves' later stages, where excess carrier concentration is low. For thinner epilayers ( 12 to $<17 \mu \mathrm{m})$, the NBE shows dominating substrate contributions, leading to very short NBE decay times. Here, the defect PL decay at room temperature shows time constants consistent with the MDP decay.

These results show that in contrast to the NBE, the MDP and defect PL decay are less sensitive to distorting influences of substrate carrier recombination and may thus be generally better suited to investigate carrier lifetimes in thin $4 \mathrm{H}-\mathrm{SiC}$ epilayers.

\section{Acknowledgment.}

This work is financially supported by the European Union (European Regional Development Fund) and by the Saxonian Government (grant no. 100249337).

\section{References}

[1] N.T. Son, X.T. Trinh, L.S. Løvlie et al., Phys. Rev. Lett. 109 (2012) 187603.

[2] P.B. Klein, R. Myers-Ward, K.-K. Lew et al., J. Appl. Phys. 108 (2010) 33713.

[3] B. Kallinger, P. Berwian, J. Friedrich, B. Thomas, J. Cryst. Growth 381 (2013) 127-133.

[4] P. Grivickas, J. Linnros, V. Grivickas, J. Mater. Res. 16 (2001) 524-528.

[5] T. Miyazawa, M. Ito, H. Tsuchida, Appl. Phys. Lett. 97 (2010) 202106.

[6] J.P. Bergman, O. Kordina, E. Janzén, phys. stat. sol. (a) 162 (1997) 65-77.

[7] P.B. Klein, J. Appl. Phys. 103 (2008) 33702. 
[8] A. Kakanakova-Georgieva, R. Yakimova, A. Henry et al., J. Appl. Phys. 91 (2002) 2890.

[9] S.G. Sridhara, L.L. Clemen, R.P. Devaty et al., J. Appl. Phys. 83 (1998) 7909-7919.

[10]L.J. Brillson, S. Tumakha, R.S. Okojie et al., J. Phys.: Condens. Matter 16 (2004) S1733.

[11] G. Feng, J. Suda, T. Kimoto, Appl. Phys. Lett. 92 (2008) 221906.

[12] S.G. Sridhara, R.P. Devaty, W.J. Choyke, J. Appl. Phys. 84 (1998) 2963.

[13] T. Kimoto, T. Hiyoshi, T. Hayashi, J. Suda, J. Appl. Phys. 108 (2010) 83721. 